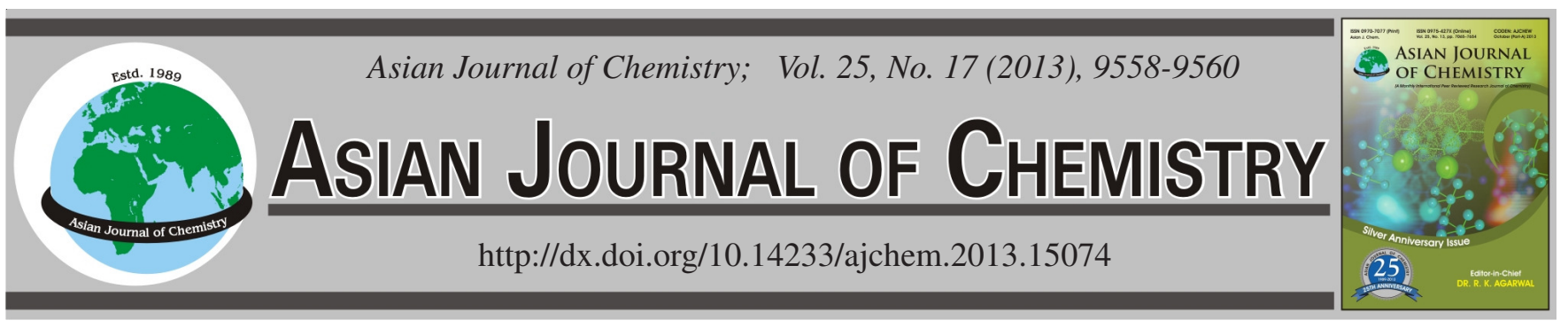

\title{
Simultaneous Determination of Danshensu, Protocatechuic Acid, Protocatechuic Aldehyde, Rosmarinic Acid and Salvianolic Acid B in Three Processing Products of White Flower Salvia miltiorrhiza by HPLC
}

\author{
Yuqin Li, Guirong You*, Rui Duan, Ke Liu and Decai Wang
}

School of Pharmacy, Taishan Medical University, Taian 271016, P.R. China

*Corresponding author: Tel./Fax: +86 538 6229751; E-mail: gryou@tsmc.edu.cn

(Received: 31 December 2012;

Accepted: 1 October 2013)

AJC-14228

\begin{abstract}
A reversed-phase high performance liquid chromatographic method was established for the simultaneous determination of five watersoluble components in three processing products of white flower Radix Salvia miltiorrhiza. The analysis was carried on a Yilite $\mathrm{C}_{18}$ column $(250 \mathrm{~mm} \times 4.6 \mathrm{~mm}, 5 \mu \mathrm{m})$. The mobile phase was a mixture of methanol and $0.5 \%$ glacial acetic acid, employing gradient elution at a flow rate of $1 \mathrm{~mL} \mathrm{~min}^{-1}$. Detection wavelength was at $286 \mathrm{~nm}$. Regression equations revealed good linear relationship between the peak areas of the compounds and their concentrations (correlation coefficients: 0.9990-0.9999). The recoveries were between 98.91 and $104.4 \%$. The results indicated that protocatechuic aicd wasn't detected in the three processed products and the rest four contents presented the fresh $<$ the wine fried $<$ the carbonizing and provided the theory foundation for pharmacy and clinic.
\end{abstract}

Key Words: Danshensu, Protocatechuic acid, Protocatechuic aldehyde, Rosmarinic acid, Salvianolic acid B, Salvia miltiorrhiza.

ᄂ _ - - - - - - - - - - - - - - - - - - - - - - - - -

\section{INTRODUCTION}

White flower $S$. miltiorrhiza (WFSM) is a variety of $S$. miltiorrhiza Bge. White flower $S$. miltiorrhiza has been grown only in mountain range and surrounding areas of Taishan of China ${ }^{1}$. The crude drugs and the processing products of roots of WFSM are widely used to for medical purpose in clinic ${ }^{2}$. The crude drugs have been used to treat palpitationzhengzhong and insomnia due to febrile disease, boils, carbuncles, pain and swelling. The processing products have been used to treat stagnation and blood stasis, angina, chest tightness, palpitation and shortness of breath ${ }^{3}$. The effective components of WFSM could be classified as lipid-soluble and water-soluble. Recent years, rescearch of water-soluble constituents in WFSM has been only focused on one or two compounds, which could not reflect the overall quality of $\mathrm{WFSM}^{4,5}$. Danshensu (DSS), protocatechuic acid (PAC), protocatechuic aldehyde (PAL), rosmarinic acid (RA) and salvianolic acid B (SAB) have been isolated and identified as the main constitutes in antiatherosclerosis, antiphlegmonosis, antioxidation and protecting myocardial damage in $\mathrm{WFSM}^{6}$. SAB can effectively restrain lipid peroxidation of the brain, liver and kidney ${ }^{7-11}$. And decoction with water is the conventional method of taking Chinese traditional medicine. Therefore, simultaneous determination of the five water-soluble constituents could control the overall quantity of WFSM.
In this report, a simple, rapid and accurate method was proposed for simultaneous determination DSS, PAC, PAL, RA and $\mathrm{SAB}$ in the three processing products of WFSM with HPLC.

\section{EXPERIMENTAL}

All separations were performed on a Shimadue LC10ATvpPlus system (Shimadue Technologies, Suzhou, China) equipped with a SPD-10A VP detector. The system was controlled by an analytical workstation. The column was a Dalian Yilite $\mathrm{C}_{18}$ reserved-phase column $(5 \mu \mathrm{m}, 250 \mathrm{~mm} \times 4.6 \mathrm{~mm})$. The sample injection volume was $20 \mu \mathrm{L}$.

Danshensu, PAC, PAL, RA and SAB were purchased from the National Institute for Control of Biological and Pharmaceutical Products of China. HPLC grade methanol was purchased from Biaoshiqi Science and techonlogy Co., Ltd. (Tianjin, China). The roots of WFSM were collected from the planting base of Taishan Medical University and identified by prof. Gao Hongli. The processing products of WFSM (Fig. 1A-C) were made by lecturer Duanrui according to the Pharmacopoeia of People's Republic of China (2010 Edition) ${ }^{12}$.

Preparation of calibration standard solutions: The stock solutions of DSS, PAC, PAL, RA and SAB were prepared with water, respectively and diluted to the appropriate concentration range (Table-1) for the establishment of calibration 


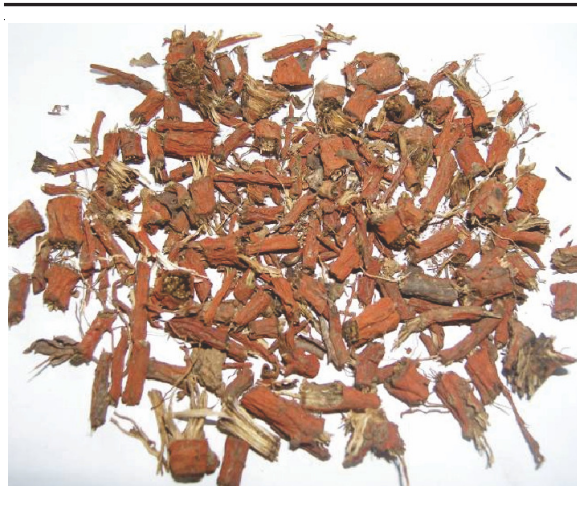

(a)

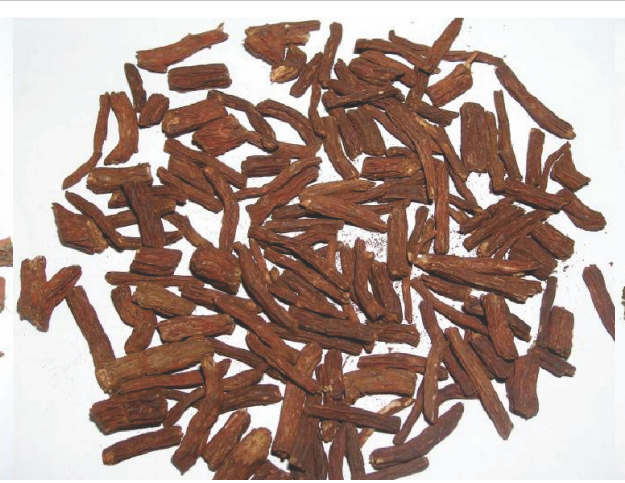

(b)

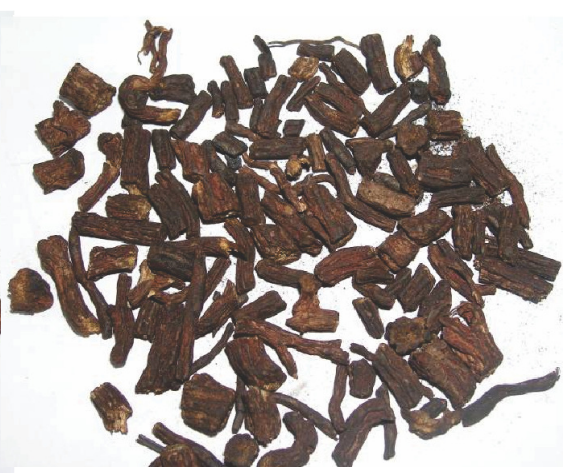

(c)

Fig. 1. Pictures of the three processing products of WFSM. (a) The crude of WFSM. (b) The wine-processing of WFSM. (c) The stir-frying of WFSM

\begin{tabular}{|c|c|c|c|c|}
\hline \multicolumn{5}{|c|}{$\begin{array}{c}\text { TABLE-1 } \\
\text { RESULTS OF REGRESSION ANALYSIS ON CALIBRATIOI }\end{array}$} \\
\hline Analytes & Regression equation $\mathrm{Y}=\mathrm{a}+\mathrm{bx}$ & Linear range $\left(\mu \mathrm{g} \mathrm{mL}^{-1}\right)$ & Correlation coefficient & Detection limit ${ }^{\mathrm{a}}\left(\mu \mathrm{g} \mathrm{mL}^{-1}\right)$ \\
\hline DSS & $\mathrm{Y}=15230 \mathrm{X}-152.82$ & $7.31-234$ & 0.9995 & 0.028 \\
\hline PAC & $Y=44550 X+198.30$ & $6.75-216$ & 0.9999 & 0.026 \\
\hline PAL & $Y=93413 X+548.45$ & $1.65-52.8$ & 0.9990 & 0.0063 \\
\hline RA & $Y=37141 X-224.48$ & $4.67-149.5$ & 0.9998 & 0.018 \\
\hline $\mathrm{SAB}$ & $Y=19435 X+398.93$ & $42.25-1352$ & 0.9999 & 0.16 \\
\hline
\end{tabular}

curves. Each calibration curve was analyzed three times with six different concentrations.

Sample preparation: The powdered (100 mesh, $2.00 \mathrm{~g}$ ) of the crude drugs of WFSM was placed in the $100 \mathrm{~mL}$ flask with plug. Then $20 \mathrm{~mL}$ water was added and lided. This mixture was oscillated for $1 \mathrm{~h}$ at $60{ }^{\circ} \mathrm{C}$ and then, decocted for $0.5 \mathrm{~h}$ at $100{ }^{\circ} \mathrm{C}$. The extraction was cooled at room temperature and centrifugalized for $5 \mathrm{~min}$ at $4000 \mathrm{rpm}$. The upper solution was collected. The residue was repeatedly extracted with the same method. The upper solutions of the twice extrations were merged and dissolved with water to $50 \mathrm{~mL}$. The method of extraction of the wine-processing and the stir-frying of WFSM were the same as above.

\section{RESULTS AND DISCUSSION}

Optimization of the separation conditions: To obtain good separation, various mobile phases, detection wavelengths and flow rates were investigated. When a Yilite $\mathrm{C}_{18}$ column was selected and detection wavelength was $286 \mathrm{~nm}$, the best resolution was obtained with a gradient elution of $0.5 \%(\mathrm{v} / \mathrm{v})$ glacial acetic acid aqueous solution (A) and methonal (B). The elution programme was well optimized and conducted as follows: the first was $30 \% \mathrm{~B}$ in the range of $0-6 \mathrm{~min}$, the second linear gradient was 30-35 \% B in the range of 10-30 min and the last one was 35-40\% B in the range of 30-40 min. Then the system was restored to initial conditions after $5 \mathrm{~min}$. The solvent flow rate was $1.0 \mathrm{~mL} \mathrm{~min}^{-1}$. The typical chromatogram for a standard mixture under the optimum conditions was shown in Fig. 2A.

Linearity, repeatability and detection limit: The linear relationships between the concentration $\mathrm{X}$ of the five analytes and the corresponding peak areas (Y) were listed in Table-1. The calibration curves exhibit excellent linear behaviour over the concentration range. The detection limits were also given in Table- 1 .
The repeatability of the peak area of DSS, PAC, PAL, RA and SAB were determined at three concentration levels $(\mathrm{n}=$ 5 ) in the ranges given above, respectively. The relative standard deviations (RSD) of the peak area of each peak were $0.47-1.57,0.79-1.70,0.26-1.59,0.22-1.69$ and $0.50-2.40 \%$ (intra-day) and $0.72-1.99,0.83-2.47,0.68-1.85,0.51-1.93$ and 0.96-2.84\% (inter-day), respectively.
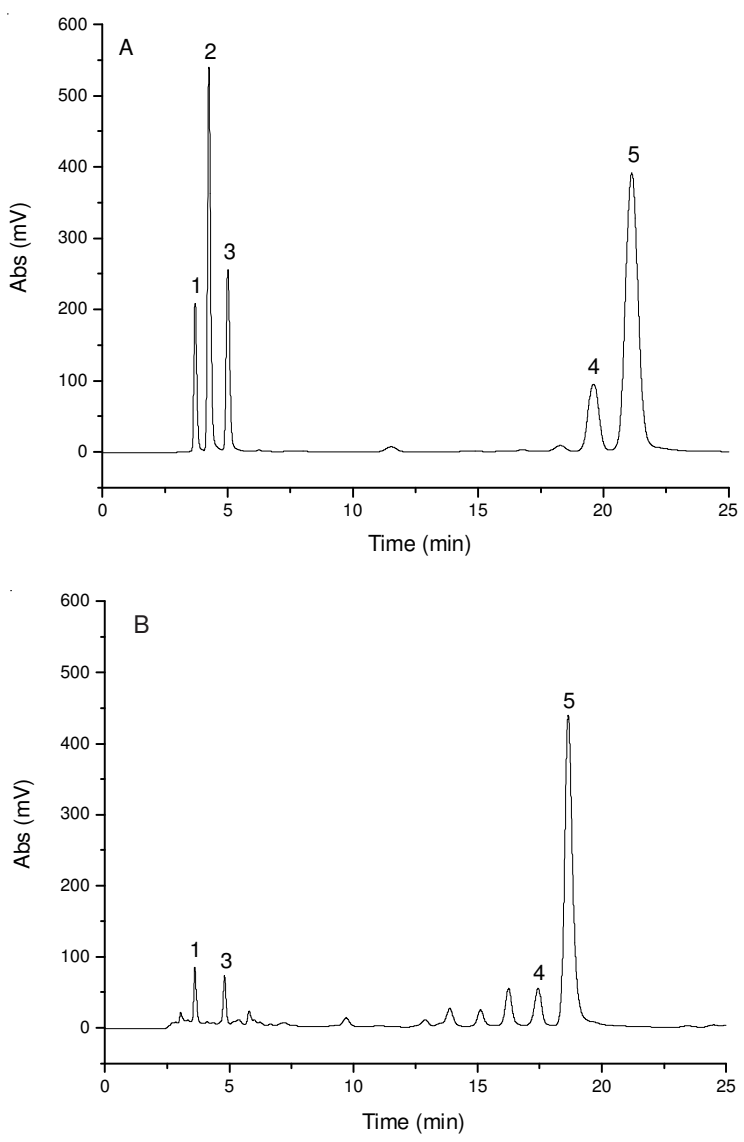

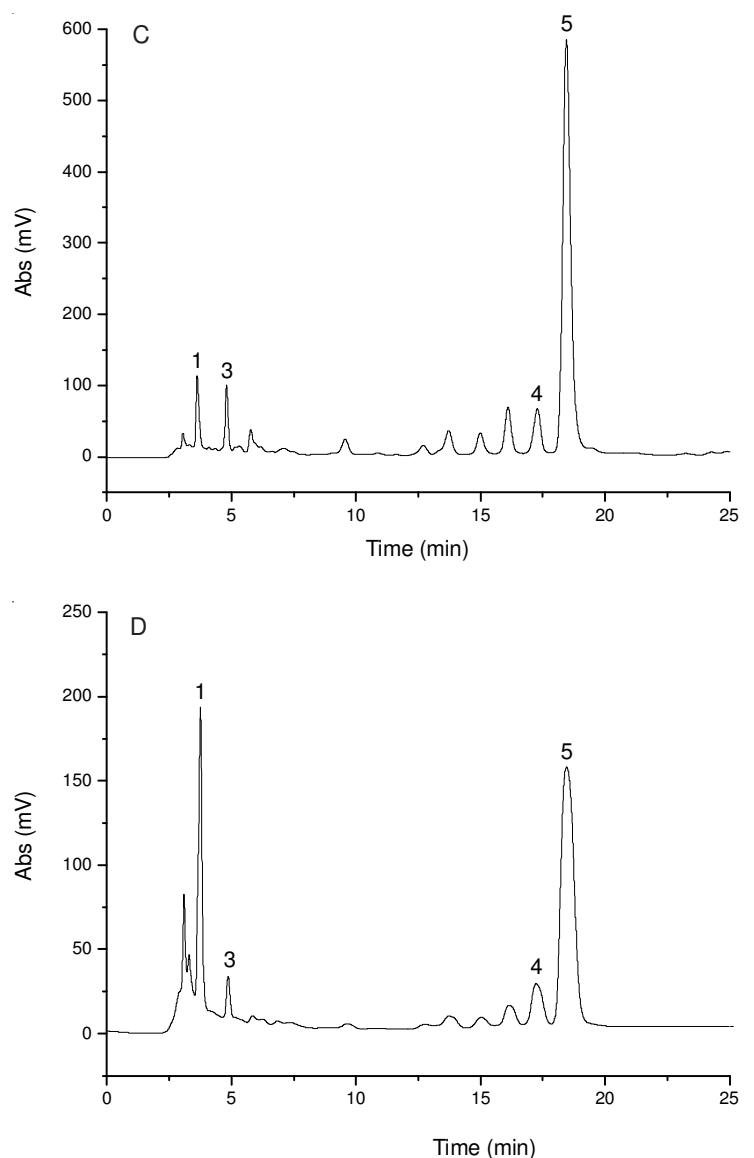

Fig. 2. HPLC chromatograms of the standards mixture solution and the samples. (A) The standard solution of 5 components. (B) The extraction of the WFSM crude. (C) The extraction of the WFSM wine-processing. (D) The extraction of the WFSM stir-frying. 1. Danshensu; 2. Protocatechuic acid; 3. Protocatechuic aldehyde; 4. Rosmarinic acid; 5. Salvianolic acid B

Application and recovery: The optimum conditions were applied to the separation and determination of DSS, PAC, PAL, RA and SAB in the three samples. The extraction of the crude was directly measured. The extraction of the wine processing was diluted three times and measured. The extraction of the stir-frying processing was diluted ten times and measured. The typical chromatograms were shown in Fig. 2B-D. The peaks were identified by comparison the migration times and by spiking the standards to the sample solution. The contents of the analytes found in the different kinds of herbs were given in Table-2.

\begin{tabular}{|c|c|c|c|}
\hline \multicolumn{4}{|c|}{$\begin{array}{c}\text { TABLE-2 } \\
\text { CONTENTS OF THE ANALYTES IN } \\
\text { THE DIFFERENT SAMPLES }(\mathrm{n}=3)\end{array}$} \\
\hline Analytes & Crude & Wine prosessing & Stir-frying processing \\
\hline DSS (\%) & 0.55 & 0.85 & 4.55 \\
\hline $\operatorname{PAC}(\%)$ & $\mathrm{ND}^{\mathrm{a}}$ & ND & ND \\
\hline PAL $(\%)$ & 0.07 & 0.11 & 0.18 \\
\hline $\mathrm{RA}(\%)$ & 0.36 & 0.34 & 0.63 \\
\hline $\mathrm{SAB}(\%)$ & 6.02 & 6.94 & 7.95 \\
\hline
\end{tabular}

From Table-2, it was found that PAC was not detected, both in the crude WFSM and the two processing products. DSS, PAL, RA and SAB were all present in the three processing products of WFSM and the contents of DSS, PAL, RA and $\mathrm{SAB}$ presented an increasing trend from the crude to the wine fried and the stir-frying.

Recovery experiments were carried out by spiking the three samples (1.000 g powder) with different amounts (low, medium and high) of standards with known contents of marker compounds and the samples were treated according to the sample preparation procedure. The results were 98.91-104.4\% for DSS, 98.72-102.8 \% for PAC, 99.82-103.5 \% for PAL, 96.07-103.1\% for RA and 96.73-105.1\% for SAB, respectively.

\section{Conclusion}

In the present study, a HPLC method was established for the simultaneous determination of the five water-soluble components in three processing products of WFSM. The method is simple, accurate and effective and can be used to control the quality of the processing products. The results indicated that protocatechuic aicd wasn't detected in the three processed products and the rest four contents presented the fresh $<$ the wine fried $<$ the carbonizing stir-frying and provided the theory foundation for pharmacy and clinic.

\section{ACKNOWLEDGEMENTS}

This work was supported by the Shandong Higher Education Institutions Foundation (J09LF30).

\section{REFERENCES}

1. C.Q. Cao, L.R. Sun, H.X. Lou and M. Ji, J. Chin. Med. Mater., 31, 938 (2008).

2. X.M. Gao, Traditional Chinese Pharmacology, Chinese Publishing House of Traditional Chinese Medicine and Pharmacology, Beijing, p. 162 (2009).

3. D.J. Ye, S.C. Zhang and H. Wu, Science of Chinese Drug Processing, People's Medical Publishing House, Beijing, p. 136 (2011).

4. Y.J. Cui, Y.Q. Li, N.N. Yang, D.W. Li, X.M. Zhao and Z.L. Xia, China Pharmacy, 19, 2592 (2008).

5. S.M. Mao, Y.L. Yan, F.X. Wang and F.J. Li, Shandong Sci., 22, 27 (2009).

6. Y.Q. Li, Y.J. Cui, X.M. Zhao, B.X. Jia and Y.X. Qi, China J. Chin. Mater. Med., 36, 1466 (2011).

7. C. Renzulli, F. Galvano, L. Pierdomenico, E. Speroni and M.C. Guerra, J. Appl. Toxicol., 24, 289 (2004).

8. A.M.J.J. Verweij-van Vught, B.J. Appelmelk, A.B.J. Groeneveld, M. Sparrius, L.G. Thijs and D.M. MacLaren, Agents Actions, 22, 288 (1987).

9. K. Van, P.M. Kok, E.S. Kalter and J. Verhoef, Oxygen Free Radicals Shock, International Workshop, pp. 79-82 (1986).

10. K.P. Van Kessel, E.S. Kalter and J. Verhoef, Agents Actions, 17, 375 (1986).

11. T. Shigematsu, S. Tajima, T. Nishikawa, S. Murad, S.R. Pinnell and I. Nishioka, Biochim. Biophys. Acta, 1200, 79 (1994).

12. Pharmacopoeia of Peoples Republic of China, 2010 ed. Part I, The Chemical Industry Publishing House, Beijing, p. 70 (2010). 